\title{
lantas parasitas do Parque Nacional Serra de Itabaiana, Sergipe, Brasil
}

\author{
Kelianne Carolina Targino de Araújo
}

Juliano Ricardo Fabricante

\begin{abstract}
Universidade Federal de Sergipe, Departamento de Biociências, Campus Universitário Prof. Alberto Carvalho, Departamento de Biociências, Laboratório de Ecologia e Conservação da Biodiversidade, Av. Vereador Olímpio Grande, s/n - Bloco D, Porto, 49.510-200, Itabaiana, Sergipe, Brasil. kelikarolina@hotmail.com
\end{abstract}

Greta Aline DetTke

Universidade Tecnológica Federal do Paraná, Herbário HCF, Via Marginal Rosalina Maria dos Santos, 1233, Área Urbanizada I,87301-899, Campo Mourão, Paraná, Brasil.

Resumo: $O$ parasitismo é considerado uma interação ecológica desarmônica interespecífica, onde uma das espécies é beneficiada (parasita) e a outra prejudicada (hospedeiro). Entretanto, as plantas parasitas são de extrema importância em ecossistemas naturais, servindo como fonte de alimento para as aves e outros animais. Pouco se sabe sobre esse grupo de plantas no estado de Sergipe. Assim, no presente estudo buscou-se responder as seguintes perguntas: (i) Quem são as espécies parasitas que ocorrem no Parque Nacional Serra de Itabaiana, SE (PARNASI)? (ii) Como as espécies estão estruturadas no local? (iii) Qual o grau de infestação dessas espécies sobre os hospedeiros? O trabalho foi desenvolvido na Serra de Itabaiana, PARNASI, SE, onde as espécies foram coletadas, herborizadas e depositadas no herbário ASE da Universidade Federal de Sergipe. Ao todo foram amostradas 10 espécies, pertencentes a quatro gêneros e três famílias. Cassytha filiformis, Phoradendron chrysocladon, Phoradendron quadrangulare e Phoradendron strongyloclados foram as espécies que apresentaram as maiores frequências no estudo da estrutura. O grau de infestação das espécies parasitas no PARNASI variou de baixo a médio e o estudo contribuiu com dois novos registros de ocorrência para o Estado de Sergipe: Psittacanthus excrenulatus e Phoradendron perrottetii.

Palavras-chave: hemiparasita, holoparasita, inventário florístico

\section{Parasitic plants of the Serra Itabaiana National Park, Sergipe, Brazil}

\begin{abstract}
Parasitism is considered an inter-specific disharmonic ecological interaction, where one species is benefited (parasite) and the other is harmed (host). However, parasitic plants are extremely important in natural ecosystems, serving as a source of food for birds and other animals. Little is known about this group of plants in the state of Sergipe. Thus, in the present study sought to answer the following questions: (i) Who are the parasitic species that occur in the Serra de Itabaiana National Park, SE (PARNASI)? (ii) How are the species structured on the site? (iii) What is the degree of infestation of these species on the hosts? The work was developed in Serra de Itabaiana, PARNASI, $\mathrm{SE}$, where the species were collected, herborized and deposited in the ASE herbarium of the Federal University of Sergipe. Altogether 10 species were sampled, belonging to four genera and three families. Cassytha filiformis, Phoradendron chrysocladon, Phoradendron quadrangulare and Phoradendron strongyloclados were the species that presented the highest frequencies in the study of the structure. The degree of infestation of parasitic species in PARNASI varied from low to medium and the study contributed with two new occurrence records for the State of Sergipe: Psittacanthus excrenulatus and Phoradendron perrottetii.
\end{abstract}

Keywords: hemiparasite, holoparasite, floristic inventory 


\section{INTRODUÇÃO}

Plantas parasitas são espécies que apresentam raízes modificadas, denominadas de haustórios, que são capazes de perfurar os tecidos dos hospedeiros e, assim, retirar parte ou totalmente sua nutrição dos mesmos (Kuijt, 1969). Elas podem ser classificadas em hemiparasitas (aquelas que retiram água e sais minerais dos hospedeiros, apresentam clorofila e fazem fotossíntese - Kuijt, 1969) ou holoparasitas (aquelas que dependem inteiramente de recursos retirados da planta hospedeira, apresentam redução do corpo vegetativo, não diferenciação de cloroplasto e consequentemente ausência de capacidade fotossintética - Kuijt, 1969).

O parasitismo é considerado como uma das interações ecológicas que afetam negativamente as espécies vegetais (Cazetta \& Galetti, 2003; Leal et al., 2006). Esse grupo de plantas pode modificar a estrutura e a dinâmica de comunidades onde estão inseridas, uma vez que reduzem a biomassa e alteram a alocação de recursos das plantas hospedeiras (Press \& Phoenix 2005; Shen et al., 2006) e interferem no balanço hídrico e de nutrientes, reduzindo as taxas de fotossíntese e respiração das plantas parasitadas (Aukema, 2003).

As espécies parasitas podem desfigurar e até suprimir totalmente os hospedeiros (Schallenberger, 2010). Árvores com alto grau de infestação estão mais susceptíveis ao ataque de agentes bióticos, a seca, ou a algum outro estresse ambiental (Tattar, 1978). Ainda, algumas parasitas também podem provocar prejuízos econômicos, pois são capazes de parasitar culturas agrícolas, chegando a matar as plantas, quando jovens, ou diminuir sua produção de maneira significativa, quando adultas (Pereira, 1998). Por outro lado, é importante destacar que a maioria das espécies parasitas são extremamente importantes para a fauna silvestre, pois servem de alimento para muitos animais, especialmente para aves (Watson, 2001; Cazetta \& Galetti, 2003).

Existem cerca de 4.880 espécies de plantas parasitas no mundo, distribuídas em 31 famílias (The Parasitic Plant Connection, 2019). No Brasil ocorrem mais de 150 espécies, distribuídas, especialmente, nas famílias Loranthaceae e Santalaceae (Arruda et al., 2012; Flora do Brasil 2020 em construção 2020).

Para o Estado de Sergipe foi encontrado apenas um estudo direcionado a esse grupo de plantas (White, 2011). Já para o Parque Nacional Serra de Itabaiana, SE (PARNASI) o que se tem são listas florísticas gerais, onde algumas espécies parasitas foram citadas (Mendes et al., 2010; Dantas et al., 2010; Silva et al., 2019).

Devido a importância do local de estudo para a conservação da biodiversidade regional e do escasso conhecimento sobre as plantas parasitas no Estado e no local, o presente estudo buscou responder as seguintes perguntas: (i) Quem são as espécies parasitas que ocorrem no PARNASI? (ii) Como as espécies estão estruturadas no local? (iii) Qual o grau de infestação dessas espécies sobre os hospedeiros?

\section{MATERIAL e MÉtodos}

\section{Área de ESTUdo}

O PARNASI $\left(10^{\circ} 25^{\prime} 15^{\prime \prime S} ; \quad 37^{\circ} 25^{\prime} 15^{\prime \prime} \mathrm{W}\right)$ possui área de 7.966 ha e abrange os municípios de Areia Branca, Campo do Brito, Itabaiana, Itaporanga d'Ajuda e Laranjeiras, no Estado de Sergipe (ICMBio, 2016). É composto pela Serra de Itabaiana, Serra do Cajueiro, Serra Comprida e Cafuz.

Está situado em uma zona de transição entre a Mata Atlântica e Caatinga (Costa, 2014). Segundo Araújo et al. (2019), o Parque é composto predominantemente pela Mata Atlântica em diferentes condições de conservação. A lista de plantas do PARNASI é composta atualmente por 828 espécies vasculares, distribuídas em 124 famílias (Silva et al., 2019). O clima na região, segundo a classificação de Köppen-Geiger, é As, tropical com verão seco e moderado excedente hídrico no inverno. Os solos predominantes variam de rasos (Neossolos Litólicos) a profundos e lixiviados (Neossolos Quartzarênicos) (Jacomine et al., 1975; Santos et al., 2013).

\section{Coleta e ANálise dos dados}

Levantamento florístico das espécies parasitas Foram feitas caminhadas (busca ativa) (Filgueiras et al., 1994) pela Serra de Itabaiana (Fig. 1 ), parte do PARNASI que abrange os municípios de Areia Branca e Itabaiana. Todas as espécies parasitas foram coletadas, herborizadas e depositadas no herbário ASE, da Universidade Federal de Sergipe, campus São Cristóvão, SE. No total, foram realizadas 30 expedições de pesquisa, com duração média de $4 \mathrm{~h}$ cada uma, durante os anos de 2018 e 2019.

A identificação das espécies foi realizada por meio de comparação com material existente no herbário ASE e por consulta à literatura específica e a especialistas. As famílias foram classificadas segundo o sistema APG IV (2016) e a grafia do nome dos autores segundo o International Plant Names Index - IPNI (2019). Por fim, todas as espécies amostradas foram classificadas em hemiparasitas ou holoparasitas. Estrutura vertical e avaliação do grau de infestação - Para o estudo da estrutura vertical e do grau de infestação das plantas parasitas foram selecionadas as 30 primeiras árvores com a presença de parasitas em três distintos pontos da área de estudo (10 em cada ponto). Cada 

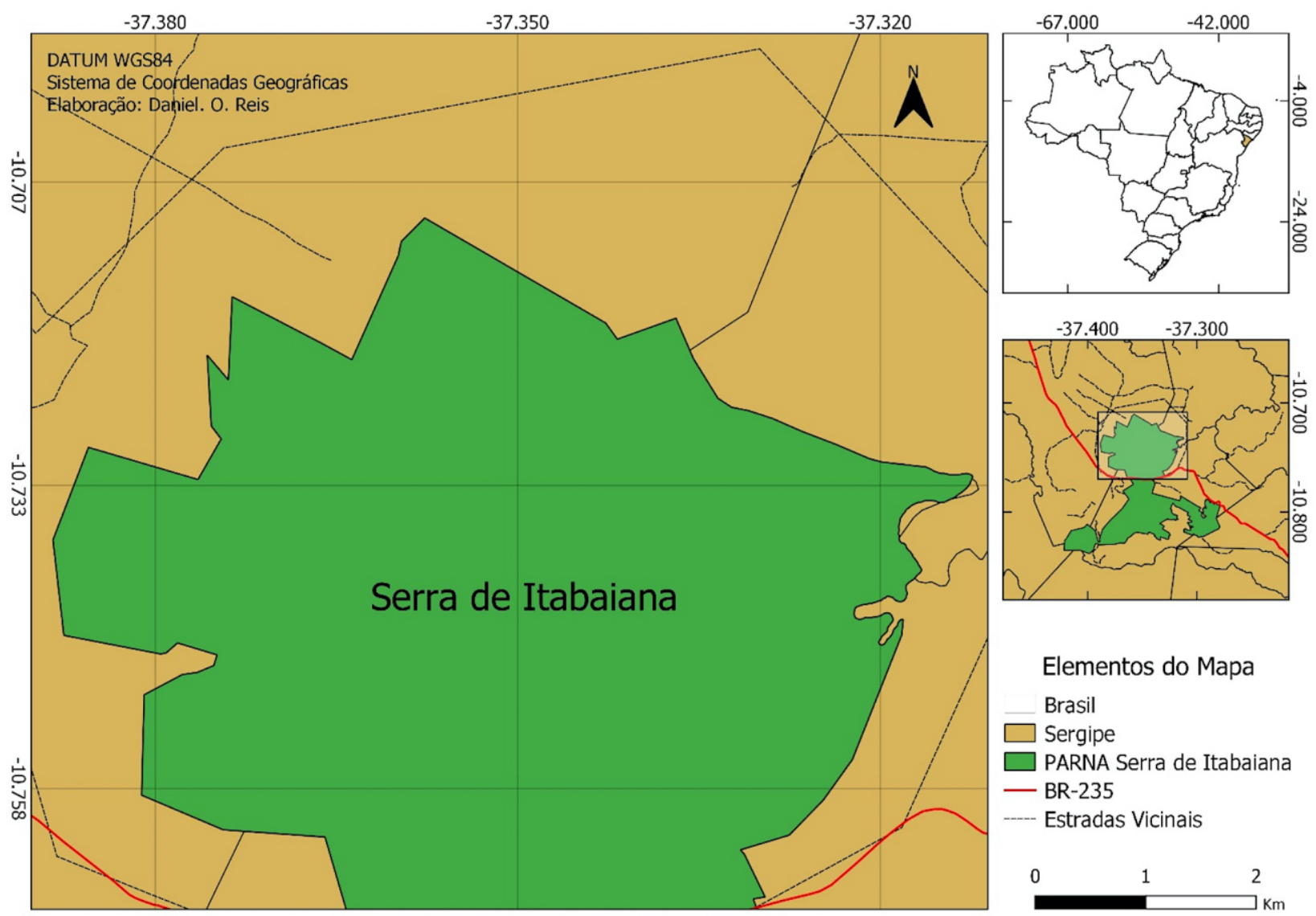

Elementos do Mapa

\section{Brasil}

$\square$ Sergipe

PARNA Serra de Itabaiana

- BR-235

--- Estradas Vicinais

0

1

2

Fig. 1. Local de estudo: Serra de Itabaina, Parque Nacional Serra de Itabaiana, Sergipe, Brasil.

indivíduo amostral (aqueles que apresentavam crescimento clonal foram considerados como um indivíduo cada agrupamento) teve o número de indivíduos de parasitas contabilizados por estrato. Para tanto, considerou-se os seguintes estratos: (i) fuste - região do caule da altura do solo até a primeira bifurcação; (ii) copa interna - região interna da copa; (iii) copa externa - regiões terminais dos galhos.

Para a avaliação da estrutura das parasitas foram calculadas a frequência absoluta e relativa sobre as plantas hospedeiras individuais $\left(F A_{i}\right.$ e $\left.F R_{i}\right)$ (adaptado de Waechter, 1998) e frequência absoluta e relativa nos estratos $\left(F A_{e}\right.$ e $F_{e}$ ) (adaptado de Kersten \& Silva, 2001).

A diversidade de parasitas foi calculada por meio do índice de Shannon-Weaver $\left(\mathrm{H}^{\prime}\right)$ (Shannon \& Weaver 1949). Já a equabilidade pelo índice de Pielou (E) (Pielou, 1977).

Cada árvore parasitada também foi avaliada quanto ao seu grau de infestação. Para tanto, considerou-se as seguintes categorias: (i) baixa incidência (até $25 \%$ da planta infestada por parasitas); (ii) média incidência (entre 25\% e $50 \%$ da planta infestada por parasitas); (iii) alta incidência (entre $50 \%$ e $75 \%$ da planta infestada por parasitas); (iv) muito alta incidência (entre $75 \%$ e $100 \%$ da planta infestada por parasitas).

\section{Resultados}

Ao todo foram amostradas 10 espécies, pertencentes a quatro gêneros e três famílias. A família com maior número de espécies foi Santalaceae com cinco táxons (50\%). Loranthaceae apresentou quatro espécies (40\%). Já Lauraceae, uma $(10 \%)$. Dentre as espécies inventariadas, nove (90\%) eram hemiparasitas e uma $(10 \%)$ holoparasita. O presente trabalho registrou pela primeira vez para o estado de Sergipe a ocorrência das espécies Psittacanthus excrenulatus Rizzini e Phoradendron perrottetii (DC.) Eichler (Tab. 1).

$\mathrm{Na}$ avaliação da estrutura da comunidade de espécies parasitas foram amostrados 216 indivíduos de seis espécies (Tab. 2). Phoradendron quadrangulare (Kunth) com 64 indivíduos $(29,6 \%)$ foi a espécie mais abundante, seguida de Cassytha filiformis L. com $56(25,9 \%)$ e Phoradendron chrysocladon A. Gray com 55 indivíduos $(25,4 \%)$. 
Tab. 1. Lista de espécies parasitas inventariadas no Parque Nacional Serra de Itabaiana, Sergipe, Brasil. Sendo: HE = Hemiparasita; HO = Holoparasita; AL - Alagoas; AM - Amapá; BA - Bahia; CE Ceará; DF - Distrito Federal; ES - Espírito Santo; GO - Goiás; MA - Maranhão; MG - Minas Gerais; MS - Mato Grosso do Sul; MT - Mato Grosso; PA - Pará; PB - Paraíba; PE - Pernambuco; PI - Piauí; PR - Paraná; RA - Roraima; RJ - Rio de Janeiro; RN - Rio Grande do Norte; RO - Rondônia; SC Santa Catarina; SE - Sergipe; SP - São Paulo; RS - Rio Grande do Sul; TO - Tocantins.

\begin{tabular}{|c|c|c|c|c|c|}
\hline Famílias & Espécies & Classificação & $\begin{array}{l}\mathbf{N}^{\circ} \text { de } \\
\text { Coleta }\end{array}$ & Figura & $\begin{array}{l}\text { Distribuição } \\
\text { Geográfica* }\end{array}$ \\
\hline Lauraceae & Cassytha filiformis L. & $\mathrm{HO}$ & FOS250 & 2 & $\begin{array}{c}\text { AL, AM, BA, CE, DF, } \\
\text { ES, GO, MA, MG, } \\
\text { MS, MT, PA, PB, PE, } \\
\text { PI, PR RA, RJ, RN, } \\
\text { RO, SE, SP, TO. }\end{array}$ \\
\hline \multirow{4}{*}{ Loranthaceae } & $\begin{array}{l}\text { Passovia podoptera } \\
\text { (Cham. \& Schltdl.) Kuijt }\end{array}$ & $\mathrm{HE}$ & KCTA20 & 3 & $B A, S E$ e $R J$. \\
\hline & $\begin{array}{l}\text { Psittacanthus dichroos } \\
\text { (Mart.) Mart. }\end{array}$ & $\mathrm{HE}$ & KCTA16 & 2 & $\begin{array}{c}A L, B A, E S, G O, M G \\
P B, P E, P R, R N, S C \text {, } \\
S E, S P, R J .\end{array}$ \\
\hline & $\begin{array}{c}\text { Psittacanthus } \\
\text { excrenulatus Rizzini }\end{array}$ & $\mathrm{HE}$ & FOS232 & 2 & BA e SE \\
\hline & $\begin{array}{c}\text { Struthanthus marginatus } \\
\text { (Desr.) Blume }\end{array}$ & $\mathrm{HE}$ & KCTA21 & 3 & $\begin{array}{c}\text { AM, BA, DF, ES, GO, } \\
\text { MA, MG, PA, PB, PE, } \\
\text { PI, RJ, RN, SE, SP, } \\
\text { TO. }\end{array}$ \\
\hline \multirow{5}{*}{ Santalaceae } & $\begin{array}{c}\text { Phoradendron } \\
\text { chrysocladon A. Gray }\end{array}$ & HE & KCTA24 & 4 & $\begin{array}{c}\mathrm{AL}, \mathrm{BA}, \mathrm{CE}, \mathrm{ES}, \mathrm{MG} \text {, } \\
\mathrm{PB}, \mathrm{PE}, \mathrm{PR}, \mathrm{RJ}, \mathrm{SC}, \\
\mathrm{SE}, \mathrm{SP} .\end{array}$ \\
\hline & $\begin{array}{l}\text { Phoradendron perrottetii } \\
\text { (DC.) Eichler }\end{array}$ & $\mathrm{HE}$ & КСТA6 & 4 & $\begin{array}{c}\text { AM, BA, DF, GO, MA, } \\
\text { MG, MS, MT, } \\
\text { PA, PB, PE, PR, RO, } \\
\text { SC, SE, SP, TO. }\end{array}$ \\
\hline & $\begin{array}{c}\text { Phoradendron } \\
\text { pteroneuron Eichler }\end{array}$ & & - & 4 & $\begin{array}{c}\mathrm{AL}, \mathrm{AM}, \mathrm{AP}, \mathrm{BA}, \mathrm{ES}, \\
\mathrm{MG}, \mathrm{MS}, \mathrm{MT}, \mathrm{PA}, \mathrm{RJ}, \\
\mathrm{SE}, \mathrm{e} S \mathrm{SP} .\end{array}$ \\
\hline & $\begin{array}{c}\text { Phoradendron } \\
\text { quadrangulare (Kunth) } \\
\text { Griseb. }\end{array}$ & HE & KCTA7 & 4 & $\begin{array}{l}\text { AL, AM, BA, CE, DF, } \\
\text { ES, GO, MA, MG, MS, } \\
\text { MT, PA, PB, PE, PI, } \\
\text { PR, RJ, RN, SC, SE, } \\
\text { SP, RS, TO. }\end{array}$ \\
\hline & $\begin{array}{c}\text { Phoradendron } \\
\text { strongyloclados Eichler }\end{array}$ & $\mathrm{HE}$ & KCTA13 & 4 & $\begin{array}{l}\text { AM, AP, BA, DF, GO, } \\
\text { MA, MG, MS, MT, PA, } \\
\text { PB, PE, PI, RJ, SE TO }\end{array}$ \\
\hline
\end{tabular}

*informações obtidas na Flora do Brasil 2020 em construção (2020).

A espécie com maior frequência individual foi C. filiformis, seguida de $P$. chrysocladon e $P$. quadrangulare. Já com maior frequência nos estratos foram $P$. chrysocladon, Phoradendron strongyloclados (Kunth) e P. quadrangulare.

A maioria dos indivíduos das espécies amostradas foram amostrados na copa externa dos hospedeiros (Tab. 3). C. filiformis, por exemplo, apresentou todos os indivíduos nessa parte do hospedeiro. Apenas duas espécies ( $P$. chrysocladon e $P$. strongyloclados apresentaram indivíduos nos três extratos considerados na avaliação (Tab. 3).

A diversidade e equitabilidade foram respectivamente de 1,48 e 0,82 . Já o grau de infestação das parasitas foi de até $25 \%$ (baixa infestação) na maioria das árvores (Tab. 4). As plantas hospedeiras que apresentaram um grau de infestação mais elevado foram aquelas que estavam sendo parasitadas por $C$. filiformis, que chegaram a ter até $50 \%$ de suas copas tomadas pela parasita (Tab. 4). 
Tab. 2. Fitossociologia das espécies parasitas amostradas no Parque Nacional Serra de Itabaiana, Sergipe, Brasil. Sendo: ni - número de indivíduos; $\mathrm{FA}_{\mathrm{i}}$ e $\mathrm{FR}_{\mathrm{i}}$ - frequência absoluta e relativa individual; $F A_{e}-F R_{e}$ frequência absoluta e relativa nos estratos.

\begin{tabular}{lccccc}
\hline \multicolumn{1}{c}{ Espécies } & $\mathrm{ni}$ & $\mathrm{FA}_{\mathrm{i}}$ & $\mathrm{FR}_{\mathrm{i}}$ & $\mathrm{FA}_{\mathrm{e}}$ & $\mathrm{FR}_{\mathrm{e}}$ \\
\hline Phoradendron quadrangulare (Kunth) & 64 & 20,7 & 20,0 & 11,5 & 22,2 \\
Cassytha filiformis L. & 56 & 27,6 & 26,7 & 9,2 & 17,8 \\
Phoradendron chrysocladon A. Gray & 55 & 24,1 & 23,3 & 13,8 & 26,7 \\
Phoradendron strongyloclados Eichler & 33 & 17,2 & 16,7 & 12,6 & 24,4 \\
Phoradendron perrottetii (DC.) Eichler & 7 & 10,3 & 10,0 & 3,4 & 6,7 \\
Psittacanthus excrenulatus Rizzini & 1 & 3,4 & 3,3 & 1,1 & 2,2 \\
\hline
\end{tabular}

Tab. 3. Distribuição vertical das espécies parasitas sobre os extratos das plantas hospedeiras no Parque Nacional Serra de Itabaiana, Sergipe, Brasil.

\begin{tabular}{lccc}
\hline \multicolumn{1}{c}{ Espécies } & Fuste & $\begin{array}{c}\text { Copa } \\
\text { Interna }\end{array}$ & $\begin{array}{c}\text { Copa } \\
\text { Externa }\end{array}$ \\
\hline Phoradendro chrysocladon A. Gray & 1 & 15 & 39 \\
Phoradendron quadrangulare (Kunth) & 0 & 20 & 44 \\
Phoradendron strongyloclados Eichler & 6 & 18 & 9 \\
Cassytha filiformis L. & 0 & 0 & 56 \\
Psittacanthus excrenulatus Rizzini & 1 & 0 & 0 \\
Phoradendron perrottetii (DC.) Eichler & 0 & 0 & 7 \\
\hline
\end{tabular}

Tab. 4. Grau de infestação (GI) das parasitas amostradas no Parque Nacional Serra de Itabaiana, Sergipe, Brasil.

\begin{tabular}{lc}
\hline \multicolumn{1}{c}{ Espécies } & Variação do GI \\
\hline Cassytha filiformis L. & $0-50 \%$ \\
Phoradendron chrysocladon A. Gray & $0-25 \%$ \\
Phoradendron perrottetii (DC.) Eichler & $0-25 \%$ \\
Phoradendron quadrangulare (Kunth) & $0-25 \%$ \\
Phoradendron strongyloclados Eichler & $0-25 \%$ \\
Psittacanthus excrenulatus Rizzini & $0-25 \%$ \\
\hline
\end{tabular}

\section{Discussão}

O número de espécies inventariadas no presente trabalho foi maior que os obtidos por outros autores (Caires, 2017; Moreira, 1997; White et al., 2011; Vasconcelos, 2015; Vasconcelos, 2016) e menor que dos estudos realizados por Dettke \& Waechte (2014) e por Reif \& Andreata (2006). Diferenças no tamanho das áreas devem explicar esses resultados. O estudo de Dettke \& Waechte (2014) abrangeu toda a região Sul e o de Reif \& Andreata (2016) todo o estado do Rio de Janeiro. O aumento no número de espécies em razão do aumento da área amostral é algo conhecido há bastante tempo (Reece et al., 2015) e parece também valer para as plantas parasitas.

Outra questão observada foi a baixa quantidade de espécies compartilhadas entre o presente estudo e pesquisas desenvolvidos em outras partes do Brasil. Com os estudos de Caires (2017) e Moreira (1997), p.e., foi apenas uma espécie em comum. Já com o trabalho desenvolvido por Dettke \& Waechte (2014), foram três. Esse fato demonstra a grande importância de cada local para a conservação das espécies parasitas.

A maior quantidade de plantas hemiparasitas amostradas nesse estudo já era esperada, uma vez elas apresentam mais representantes quando comparado com as holoparasitas (The Parasitic Plant Connection, 2019). Outros estudos obtiveram resultados semelhantes (Caires, 2017; Moreira, 1997; White et al., 2011; Vasconcelos, 2015; Vasconcelos, 2016; Dettke \& Waechte, 2014; Reif \& Andreata 2016). 

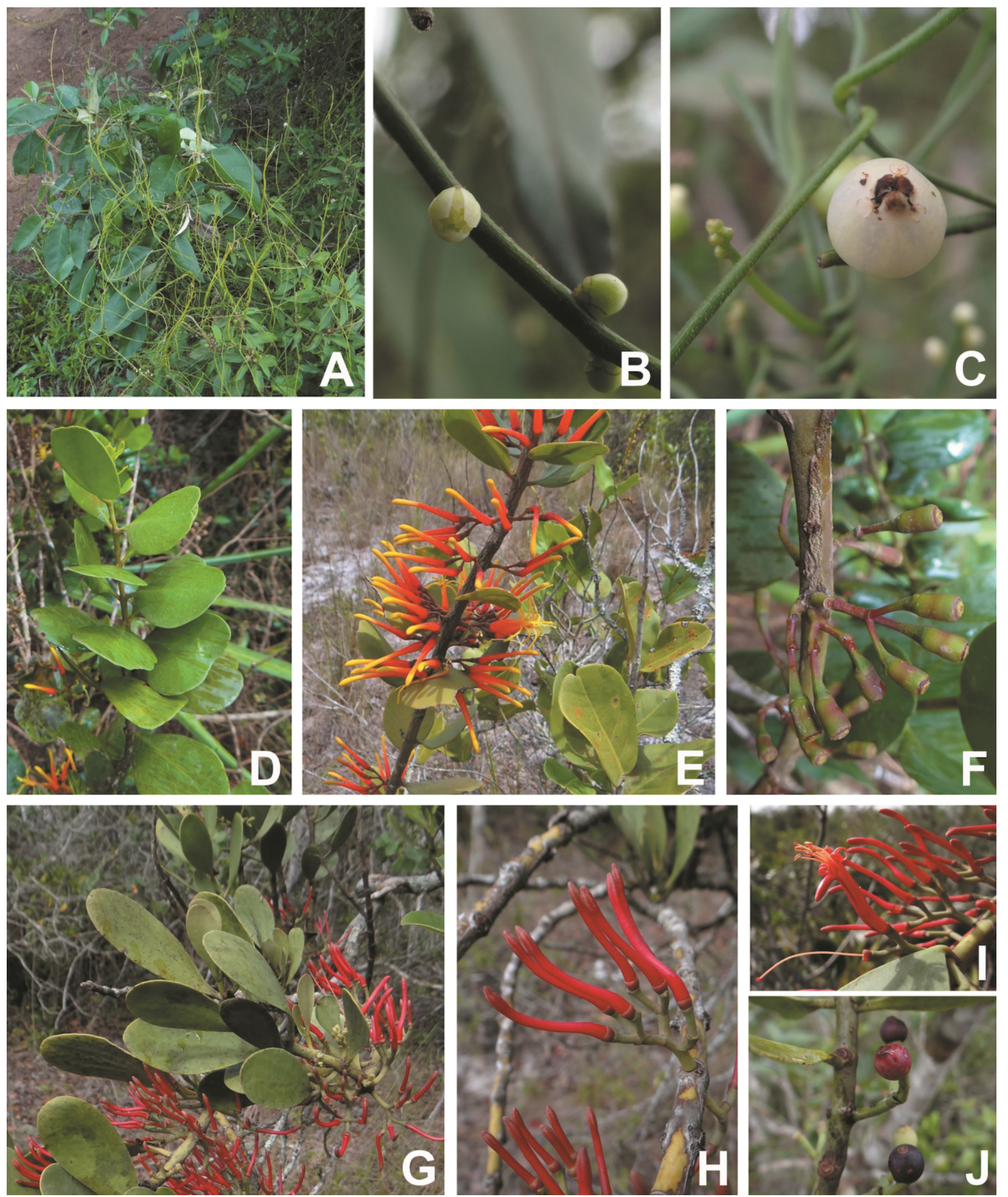

Fig. 2. Cassytha filiformis L. (Lauraceae). A. Ramos. B. Flores. C. Fruto maduro (A-C de Silva 250). Psittacanthus dichroos (Mart.) Mart. (Loranthaceae). D. Ramo. E. Flores. F. Frutos imaturos (D-F de Araújo 16). Psittacanthus excrenulatus Rizzini (Loranthaceae). G. Ramos floridos. H. Botões florais. I. Flor. J. Frutos maduros (G-J de Silva 232).

Não foram encontrados estudos com parasitas que calcularam índices de diversidade, impedindo, portanto, estabelecer se o valor obtido no presente estudo é baixo ou alto. Porém, o valor de 0,82 da equitabilidade é considerado elevado, indicando uma homogeneidade na distribuição dos indivíduos entre as espécies.

Santalaceae, a família com maior número 

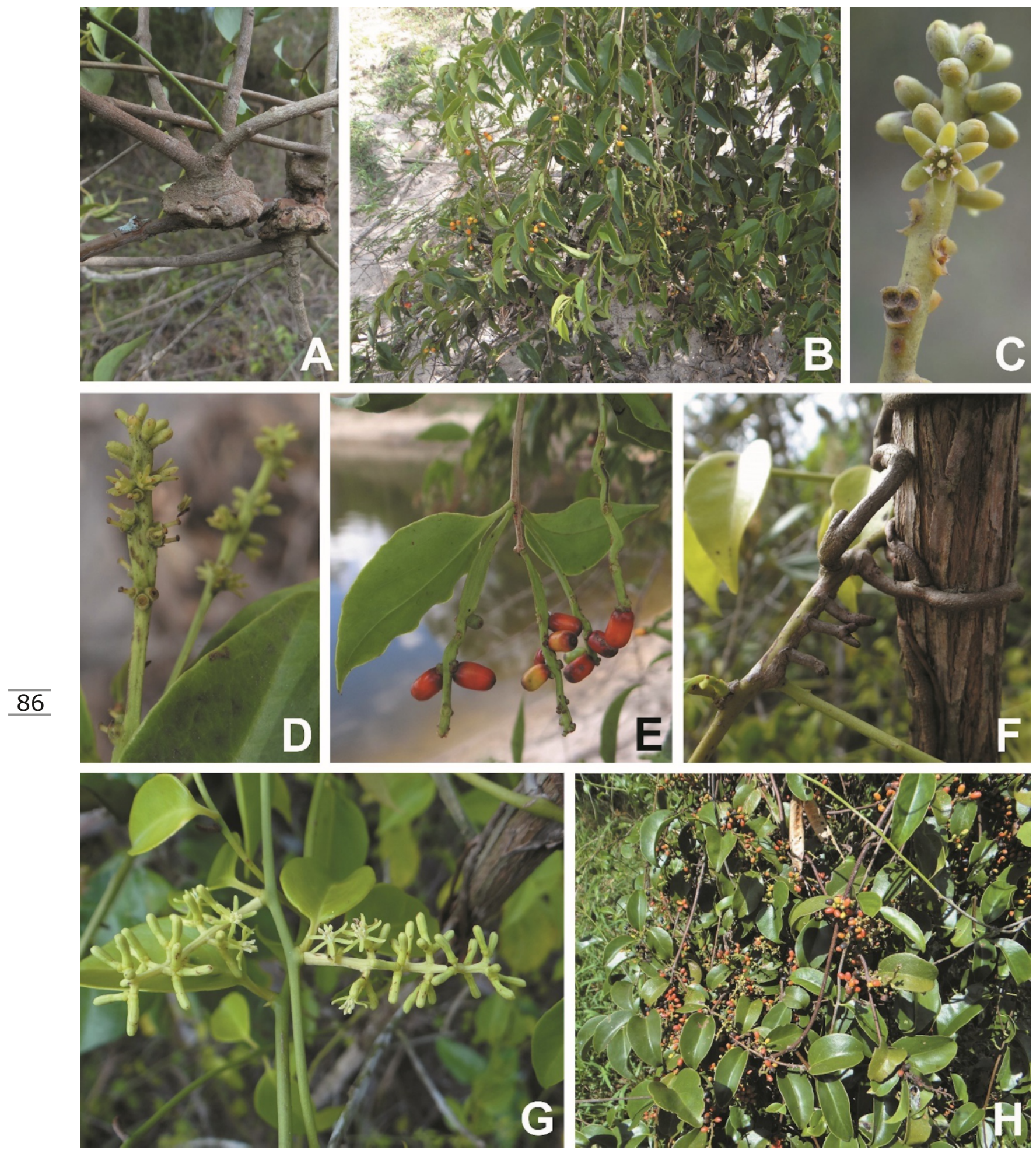

Fig. 3. Passovia podoptera (Cham. \& Schltdl.) Kuijt (Loranthaceae). A. Haustório primário. B. Ramos. C. Inflorescência estaminada. D. Inflorescência pistilada. E. Frutos maduros (A-E de Araújo 20). Struthanthus marginatus (Desr.) Blume (Loranthaceae). F. Haustórios secundários. G. Inflorescências. H. Ramos com frutos maduros (F-H de Araújo 12).

de espécies no presente estudo, apresentou apenas um gênero: Phoradendron. Segundo Dettke \& Waechter (2014), esse gênero é o que apresenta maior diversidade dentro da família, chegando a possuir 60 espécies distribuídas em todos os domínios fitogeográficos brasileiros. No Brasil, as espécies amostradas dessa família estão presentes em quase todos os domínios fito- 

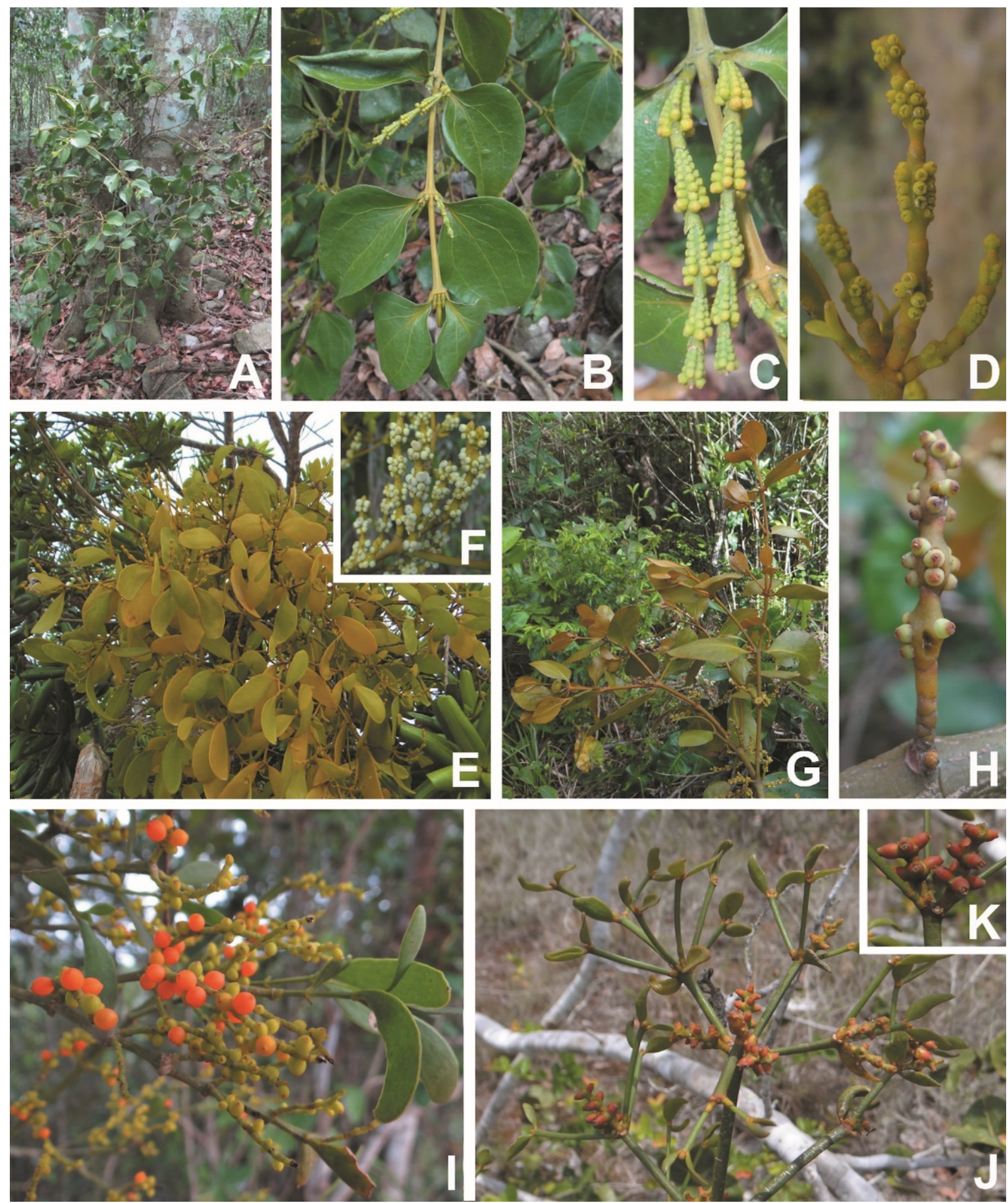

Fig. 4. Phoradendron chrysocladon A. Gray (Santalaceae). A. Indivíduo. B. Ramos. C. Frutos imaturos (A-C de Araújo 24). Phoradendron perrottetii (DC.) Eichler (Santalaceae). D. Inflorescências. E. Indivíduo. F. Frutos maduros (D-F de Silva 246). Phoradendron pteroneuron Eichler (Santalaceae). G. Ramos. H. Frutos imaturos (G-H de Araújo 12) I. Phoradendron quadrangulare (Kunth) Griseb. (Santalaceae), ramos com frutos imaturos e maduros (I de Araújo 7). Phoradendron strongyloclados Eichler (Santalaceae). J. Ramos. K. Frutos imaturos (J-K de Araújo 13).

geográficos, com destaque para $P$. perrottetii, que foi um dos novos registros para o estado de
Sergipe. Até então, ela só havia sido registrada no Nordeste nos Estados do Maranhão, Paraíba, 
Pernambuco e Bahia (Flora do Brasil 2020 em construção 2020).

Já Loranthaceae, a segunda família com maior número de espécies também apresenta grande importância, por servir como fonte de alimento para diversas espécies de aves (Tainter, 2002) e possuir propriedades medicinais (Siqueira et al., 2016). As espécies dessa família ocorrem em diferentes ecossistemas (Norton $\&$ Carpenter, 1998). Além disso, é considerada a maior família entre as parasitas (Harris, 1992), apresentando 73 gêneros ocorrentes nos trópicos do Velho e Novo mundo e alguns ocorrendo em regiões temperadas (Nickrent et al., 2010).

No Brasil, Psittacanthus dichroos (Mart.) Mart. e Struthanthus marginatus (Desr.) Blume, ambas amostradas no presente trabalho, ocorrem em todas as regiões da federação (Flora do Brasil 2020 em construção, 2020). Vale ressaltar que o gênero Struthanthus possui cerca de 60 espécies com ocorrência na América tropical, sendo 40 delas originárias do Brasil (Rizzini, 1968).

A espécie $S$. marginatus é bastante comum no território brasileiro e apresenta propriedades medicinais reconhecidas pela farmacologia brasileira. Segundo Siqueira et al. (2016), a espécie apresenta eficácia na cura de infecções respiratórias. Já o táxon $P$. excrenulatus até o momento apresentava uma distribuição restrita ao Estado da Bahia (Flora do Brasil 2020 em construção, 2020). Esse fato reforça a relevância do presente trabalho.

Parte das espécies que se destacaram no estudo estrutural são generalistas quanto aos hospedeiros (Caires \& Proença, 2008; Nickrent, 2004). Tal fato deve explicar os resultados obtidos.

Apesar dos efeitos negativos que as parasitas podem causar aos seus hospedeiros, considera-se que na área de estudo estas espécies são muito importantes uma vez que servem de recurso alimentar para a fauna local, como por exemplo, para espécies de pássaros frugívoros das famílias Ramphastidae e Cotingidae (ICMBio, 2016) ou para aves polinizadoras como Prodotiscus regulus Sundevall 1850 e Arachnothera longirostris Latham 1790 (Watson, 2001).

Os resultados do grau de infestação corroboram com os encontrados por White et al. (2011), onde também houve uma predominância de baixa infestação nas plantas hospedeiras. Além disso, as poucas hospedeiras que apresentaram um grau de infestação de média incidência, estavam sendo parasitadas por $C$. filiformis.

A maior predominância de espécies hemiparasitas na parte externa das copas deve estar relacionada a questões fisiológicas. Nessa região há uma maior incidência de radiação solar e é notório que esse recurso é indispensável para a realização da fotossíntese (Lousã et al., 2007). Além disso, Cazetta \& Galetti (2007) afirmam que os locais utilizados pelas aves como poleiros, podem influenciar na distribuição das parasitas sobre as plantas hospedeiras. Esse segundo argumento explicaria por que $C$. filiformis, uma holoparasita, também apresentou o mesmo comportamento das hemiparasitas.

Segundo Radomiljac (1998), o substrato ao qual a planta parasita está aderida pouco influencia na germinação da semente, contudo, galhos de maior diâmetro influenciam positivamente no estabelecimento delas. Nossos resultados, porém, refutaram essa afirmação, já que a maioria dos indivíduos foram amostrados na copa externa dos hospedeiros.

Nossos dados demonstraram: (i) a ocorrência de 10 espécies parasitas na Serra de Itabaiana, PARNASI, SE, dentre as quais nove hemiparasitas e um holoparasita; (ii) que Cassytha filiformis, Phoradendron chrysocladon, Phoradendron quadrangulare e Phoradendron strongyloclados foram as espécies que apresentaram as maiores frequências e que a maioria das espécies foram amostradas na copa externa dos hospedeiros; (iii) a maioria das parasitas apresentou índices de infestação de até $25 \%$. Além disso, nosso estudo também contribuiu para o relato de ocorrência de duas novas espécies para o estado de Sergipe: Psittacanthus excrenulatus e Phoradendron perrottetii.

\section{ReferênCIAS}

APG IV. 2016. An update of the Angiosperm Phylogeny Group classification for the orders and families of flowering plants: APG IV. Bot. J. Linn. Soc. 181: 1-20.

Araújo, K. C. T., J. L. Santos \& J. R. Fabricante. 2019. Epífitas vasculares do Parque Nacional Serra de Itabaiana, Sergipe, Brasil. Biotemas. 31: 31-29.

Arruda, R., R. F. Fadini, L. N. Carvalho, K. Del-Claro, F. A. Mourão, C. M. Jacobi, G. S. Teodoro, E. Vandenberg, C. S. Caires, \& G. A. Dettke. 2012. Ecology of neotropical mistletoes: an important canopy- dwelling component of Brazilian ecosystems. Acta Bot. Bras. 26: 264-274.

Aukema, J. E. 2003. Vectors, viscin, and Viscaceae: mistletoes as parasites, mutualists and resources. Frontiers in Ecol. and the Env. 1: 212-219.

Caires, C. S. \& C. E. B. Proença. 2008. Levantamento preliminar dos hospedeiros de Dendrophthora e Phoradendron (Santalaceae) no Distrito Federal, Brasil. Hering. 2: $11-22$. 
Caires, C. S. 2017. Flora das cangas da Serra dos Carajás, Pará, Brasil: Santalaceae. Rodriguésia. 68: 1139-1149.

Cazetta, E. \& M. Galetti. 2003. Ecologia das ervas-de-passarinho. Ci. Hoje. 33: 72-74.

Cazetta, E. \& M. Galetti. 2007. Frugivoria e especificidade por hospedeiros na erva-depassarinho Phoradendron rubrum (L.) Griseb. (Viscaceae). Rev. Bras. Bot. 30: 345351, 2007.

Costa, C. C. 2014. Parque Nacional Serra de Itabaiana-SE: realidade e gestão. Remoa. 13: 3933-3951.

Dantas T. V. P., J. E. Nascimento-Júnior, A. S. Ribeiro \& A. P. N. Prata. 2010.Florística e estrutura da vegetação arbustivo-arbórea das Areias Brancas do Parque Nacional Serra de Itabaiana/Sergipe, Brasil. Rev. Bras. Bot. 33: 575-588.

Dettke, G. A. \& J. L. Waechter. 2014. Estudo taxonômico das ervas-de-passarinho da Região Sul do Brasil: II. Viscaceae (Phoradendron). Rodriguésia. 65(4): 955-985.

Filgueiras, T. S., P. E. Nogueira, A. L. Brochado \& G. F. Guala. 1994. Caminhamento - um método expedito para levantamentos florísticos qualitativos. Dir. Geo. 39-43.

Harris, R. W. 1992. Arboriculture: integrated management of landscape trees, shrubs and vines. New Jersey, Prentice-Hall.

Flora do Brasil. 2020. Disponível em:< http:// floradobrasil.jbrj.gov.br/reflora/listaBrasil/ ConsultaPublicaUC/ConsultaPublicaUC.do\#CondicaoTaxonCP>. Acesso em 14 de jun. 2020.

ICMBio. 2016. Plano de Manejo Parque Nacional Serra de Itabaiana.

IPNI. 2019. Disponível em: <https://www.ipni.org/>. Acesso em 06 de ago. 2019.

Jacomine, P. K. T., J. O. Montenegro, M. R. Ribeiro \& R. A. Formiga. 1975. Levantamento exploratório-reconhecimento de solos do estado de Sergipe. Recife, EMBRAPA, Centro de Pesquisas Peclológicas, ilus. (Brasil. EMBRAPA. Centro de Pesquisas Pedológicas. Bol. Tec. 36). Brasil, SUDENE, DRN. Divisão de Recursos Renováveis.
Kersten, R. A. \& S. M. Silva. 2001. Composição florística e estrutura do componente epifítico vascular em Floresta da planície litorânea na Ilha do Mel, Paraná, Brasil. Rev. Bras. Bot. 24: 213-226.

Kovach, W. L. 2005. MVSP - A multivariate statistical package for windows, ver. 3.1. Pentraeth, K.C.S.

Kuijt, J. 1969. The Biology of Parasitic Flowering Plants. University of California Press, Berkeley and Los Angles.

Kuijt, J. 2003. Monograph of Phoradendron. Syst. Bot. Mono. 66: 1-643.

Leal, L., W. M. Bujokas \& D. Biondi. 2006. Análise da Infestação de Erva-de-Passarinho na arborização de ruas de Curitiba-PR. Flo. 36: 323-330.

Lousã, M., A. Monteiro, D. Espírito Santo, E. Sousa \& J. C. Costa. 2007. Módulo de Botânica Manual de Teóricas e Práticas. Lisboa, Inst. Sup. Agro. 144 p.

Mendes, K., P. Gomes \& M. Alves. 2010, Floristic inventory of a zone of ecological tension in the Atlantic Forest of Northeastern Brazil. Rodriguésia. 61: 669-676.

Moreira, B. A. \& C. M. Rizzini. 1997. As famílias Loranthaceae e Viscaceae da APA de Maricá, Rio de Janeiro, Brasil. Acta. Bot. Bras. 11: 1-8.

Müller-Dombois, D. \& H. Ellenberg. 1974. Aims and methods of vegetation ecology. New York, John Wiley \& Sons.

Nickrent, D. L. \& L. J. Musselman. 2004. Introduction to parasitic flowering plants. PHI. DOI: http://dx.doi.org/10.1094/PHI-I2004-0330-01

Norton, D. A. \& M. A. Carpenter. 1998. Mistletoes as parasites: host specificity and speciation. TREE. 13: 101-105.

Pereira, W. 1998. Prevenção e Controle da Parasita Cuscuta em áreas cultivadas com hortaliças. Brasília, Embrapa Hortaliças. 8 p. (Comunicado Técnico, 9).

Pielou, E. C. 1977. Mathematical ecology. New York/London/Sydney/Toronto, Wiley-Interscience Publ.

Press, M. C. \& G. K. Phoenix. 2005. Impacts of parasitic plants on natural communities. New. Phyto. 166: 737-751. 
Radomiljac, A. M. 1998. The influence of pot host species, seedling age and supplementary nursery nutrition on album Linn. (Indian sandawood) plantation stablishment within the Ord River Irrigation Area, Western Australia. For. Ecol. Manag. 102: 193201.

Reece, J. B., A. Steven, L. A. Wasserman, M. L. Urry, P. V. Cain, R. B. J. Minorsky. 2015. Biologia de Campbel. 10 ed., Porto Alegre, Artmed.

Reif, C. \& R. H. P. Andreata. 2006. Sinopse de "Ervas-de-Passarinho" do estado do Rio de Janeiro, Brasil. Pesqu. Bot. 255-274.

Rizzini, C. T. 1968. Flora Ilustrada Catarinense. Itajaí, Santa Catarina.

Rizzini, C. T. 1952 Phthirusae brasiliae terrarumque adiacentium. Dusenia. 3: 451-462.

Santos, H. G., P. K. T. Jacomine, L. H. C. Anjos, V. A. Oliveira, J. F. Lumbreras, M. R. Coelho, J. A. Almeida, T. J. F. Cunha \& J. B. Oliveira. 2013. Sistema Brasileiro de Classificação de Solos. $3^{a}$ ed., Brasília, Embrapa.

Sargent, S. 1995 . Seed fate in a tropical mistletoe: the importance of host twig size. Func. Ecol. 9: 127-204.

Schallenberger, L. S., A. J. Araújo, M. N. Araújo, L. J. Deiner \& G. O. Machado. 2010. Avaliação da condição de árvores urbanas nos principais parques e praças no município de Irati-PR. Revsbau. 5: 105123.

Shannon, C. \& W. Weaver. 1949. The Mathematical Theory of Communication. Urbana, Uni.Ill.Press.

Shen, H., W. Ye, L. Hong, H. Huang, $Z$. Wang, X. Deng, Q. Yang \& Z. Xu. 2006. Progress in parasitic plant biology: Host selection and nutrient transfer. Plant Bio. 8: 175-185.

Silva, A. C. C., E. V. S. Oliveira, M. Alves, M. C. V. Farias, A. C. Mota, C. A. S. Souza \& A. P. N. Prata. 2019. Lista atualizada da flora vascular do Parque Nacional (PARNA) Serra de Itabaiana, Sergipe, Brasil. Pesq. Ens. Ciênc. Exat. Nat. 3: 40-67.
Siqueira, A. M., I. T. S. Rogério, B. C. Esteves, L. M. Chedier, A. L. Macedo \& D. S. Pimenta. 2016. Chemical interaction between Struthanthus marginatus (Desr.) Blume and two different hosts. Eletronic J. of Pharmacy. 13: 212-219.

Souza, V. C. \& H. Lorenzi. 2005.Botânica Sistemática: guia ilustrado para identificação das famílias Angiospermas da flora brasileira, baseado na APGII. Nova Odessa, Inst. Plant. de Est. da Flora. 640 p.

Tainter, F. H. 2002. What does mistletoes have to do with Christmas? Feature Story. St. Paul: American Phytopathological Society. Disponível em: <https://www.apsnet.org/ edcenter/apsnetfeatures/Pages/Mistletoe.aspx>. Acesso em: 22 de out. 2019.

Tattar, T. A. 1978. Diseases of Shade Trees. New York: Academic.

The Parasitic Plant Connection. 2019. Disponível em: <https://parasiticplants.siu.edu/ $>$. Acesso em: 05 de ago. 2019.

Vasconcelos, G. C. L. \& J. I. M. Melo. 2016. Flora do Parque Nacional do Catimbau, PE, Brasil: Loranthaceae. Hoehnea. 43: 317323.

Vasconcelos, G. C. L., C. S. Caires \& J. I. M. Melo. 2015. Flora da Paraíba, Brasil: Santalaceae R.Br. Iheringia. 70: 203-215.

Waechter, J. L. 1998. O epifitismo vascular em uma floresta de restinga do Brasil subtropical. Ver. Ciênc. Nat. 20: 43-66.

Watson, D. M. 2001. Mistletoe - A Keystone resource in forests and wood lands worldwide. Annual Rev. Ecol. Syst. 32: 219-249.

White, B. L. A., A. S. Ribeiro, L. A. S. White \& J. E. Nascimento-Júnior. 2011. Análise da ocorrência de erva-de-passarinho na arborização da Universidade Federal de Sergipe, Campus São Cristóvão. Floresta. 41: 1-8. 